\title{
Passive, In-Home Gait Measurement Using an Inexpensive Depth Camera: Initial Results
}

\author{
Erik E. Stone and Marjorie Skubic \\ Center for Eldercare and Rehabilitation Technology \\ Department of Electrical and Computer Engineering \\ University of Missouri \\ Columbia, MO, USA \\ ees6c6@mizzou.edu, skubicm@missouri.edu
}

\begin{abstract}
In-home gait measurement results from the apartments of seven older adults obtained using an environmentally mounted depth camera, the Microsoft Kinect, are presented. Previous work evaluating the use of the Kinect for in-home gait assessment in a lab setting has shown the potential of this approach. In this work, a single Kinect sensor and computer have been deployed in five apartments, two of which contain multiple residents, in an independent living facility for older adults. Data collected in the five apartments, along with techniques for generating automated gait measurements from the data, are presented.
\end{abstract}

Keywords - gait; Kinect; fall risk; passive monitoring;

\section{INTRODUCTION}

$\mathrm{R}$ ESEARCH has shown the importance of measuring a person's gait [1] and that the parameters which describe locomotion are indispensible in the diagnosis of frailty and fall risk [2]. Studies have indicated that changes in gait parameters may be predictive of future falls and adverse events in older adults [3-6] and may precede cognitive impairment [7]. However, current methods for measuring gait, such as observation by a clinician or evaluation in a physical performance lab, often lead to sparse, infrequent assessments and may not be representative of a person's true functional ability [8]. Systems capable of measuring gait on a continuous basis during normal daily activity could provide invaluable information for purposes ranging from automated fall risk assessment to early detection of illness.

A number of technologies exist or are being developed for continuous gait monitoring outside of clinical and laboratory settings [8-12]. These technologies range from wearable accelerometer based devices to arrays of passive infrared motion sensors in the home. In [8], researchers were able to show that in-home walking speeds were associated with several neuropsychological and motor performance tests and that they allowed the calculation of previously unattainable metrics of physical function.

Based on responses from older adults [13], an ideal monitoring system would be unobtrusive and not inconvenience the patient. Environmentally mounted vision sensors address this concern. In addition, vision sensors offer the precision necessary to measure detailed gait parameters such as stride time and stride length, which have been shown to be important to fall risk assessment, without the need for wearable devices. Finally, research has indicated that privacy concerns of older adults to visionbased monitoring systems can be addressed by use of appropriate privacy preserving processing techniques [14].

Recently, Microsoft released the Kinect sensor for their Xbox gaming system. The sensor uses a pattern of actively emitted infrared light in combination with an image sensor to obtain a depth image that is generally invariant to ambient lighting. The sensor offers a single, low cost sensor device that allows for a three dimensional representation of the environment. Earlier work looked at developing algorithms for measuring gait parameters from the raw depth imagery of the Kinect sensor and validating the measurements against a Vicon marker-based motion capture system in a laboratory setting [11]. This evaluation showed good agreement between the two systems.

This paper presents initial results of monitoring the gait of seven older adults, living in an independent living facility, continuously, in their homes, using a Kinect sensor. Preliminary data is presented along with a methodology for computing average gait parameters for the residents. Potential future work and challenges involved in capturing the data are discussed.

\section{SYSTEM OPERATION}

The Kinect sensor and a computer were deployed in the apartments of seven elderly residents in an independent living facility with the goal of assessing and monitoring their gait. Figure 1 shows the Kinect sensor as mounted in one apartment. The Kinect is placed on a small shelf below the ceiling (height 9 feet), above the front door. For convenience to the resident, the computer is placed in a cabinet above the refrigerator. The arrangement has proven to be quite unobtrusive to residents, with some indicating that they do not notice the equipment after a short period of time.

The Microsoft Kinect SDK, and the skeletal tracking it provides, is not used. Instead, the raw disparity values from the Kinect depth stream are processed as described in [11], with functionality added to allow the system to operate in dynamic environments and track segmented people. The main reason for not using the Kinect SDK is the limited 


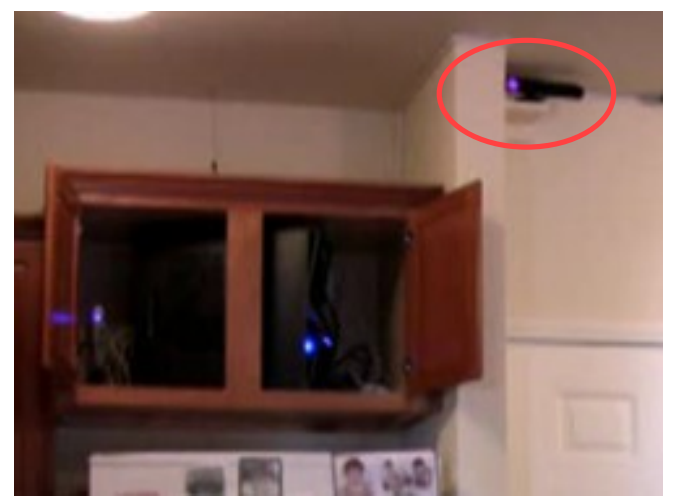

Figure 1: Kinect system deployed in an apartment in an independent living facility. The Kinect sensor is seated on a small shelf mounted close to the ceiling. A computer is placed in the cabinet above the refrigerator.

range of the skeletal tracking, approximately 1.5 to 4 meters from the Kinect. This range, combined with the positioning of the Kinect, is insufficient to capture walking sequences from beginning to end in many areas of the apartments, especially the larger ones, whereas the current approach has been shown to work at distances of up to 8 meters from the Kinect. Furthermore, the accuracy and reliability of the skeletal model provided by the SDK, in the usable range, has yet to be validated for gait measurement, whereas the accuracy and reliability of the current approach has been validated with good results.

Walking sequences are identified from the path history of people tracked by the system. A set of criteria including path straightness, speed, duration, and distance are used to extract suitable walking sequences from the path histories. This is done online in real-time. Due to issues such as occlusion and bad segmentation, stride parameters cannot be extracted for every walking sequence. For this analysis, quality walks are defined as those for which a minimum of six steps were extracted which also met three screening criteria used to eliminate possibly invalid step sequences: 1 - the steps were extracted in the correct temporal order (left, right, left, right, etc.), 2- the maximum amplitude of the correlation coefficient time series did not exceed 90 (see [11]), 3- the difference between the maximum and minimum stride times was less than half the mean stride time.

\section{RESULTS AND ANALYSIS}

The Kinect gait system has been deployed in five apartments in the independent living facility with the goal of deploying a total of ten systems for two years. Of the five apartments, three had a single resident while two had two residents. Ages of the residents range from 75 to 95 and four of the seven residents are male. For this analysis, data from the three week period November $3^{\text {rd }}$ through November $23^{\text {rd }}$, 2011, was used. Two of the residents used a walker at some point during this time period.

\section{A. Apartment 1}

Figure 2 provides visualizations of the walk data from (a)

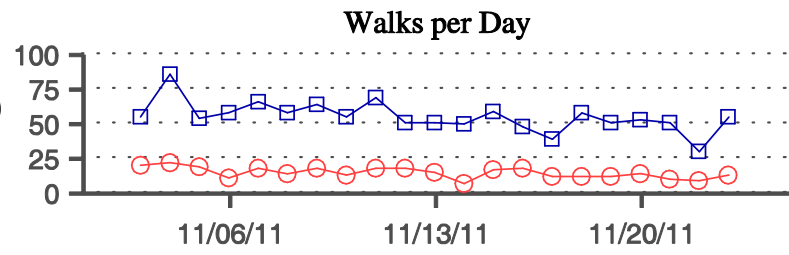

(b)

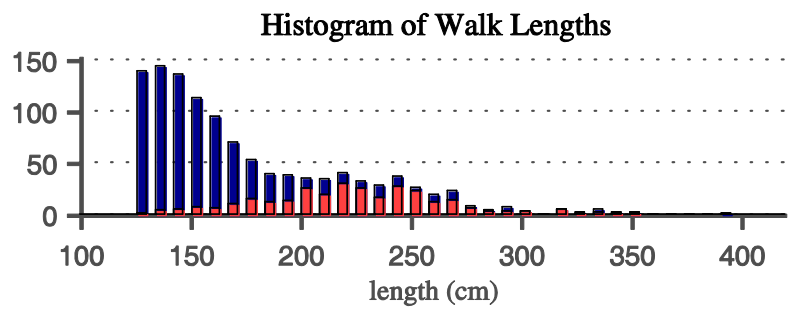

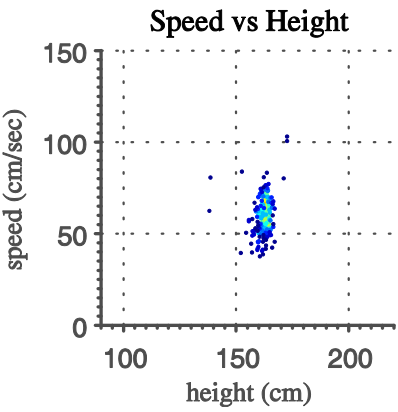

(c)

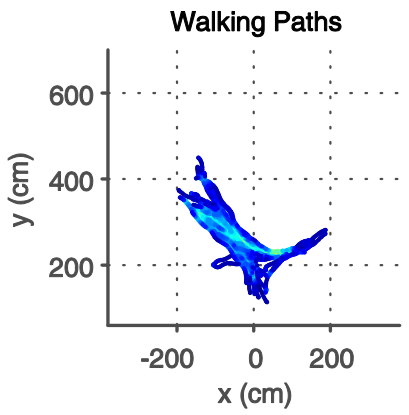

(d)
Figure 2: Data from Apartment 1. (a) Plot of the number of identified walks per day: all (blue square), quality (red circle). (b) Histogram of the walk lengths: all (blue), quality (red). (c) Density plot of speed vs. height for the quality walks. (d) Density plot of the paths of the quality walks.

Apartment 1. During the three week period, a total of 1140 walks were identified in the apartment. Of those, 297 were determined to be quality (as defined at the end of Section II). As can be seen in Figure 2(a), the Kinect system identified between 29 and 85 total walks per day and between 8 and 20 quality walks per day. The histogram of walk lengths in 2(b) illustrates not only the difference in the length distributions of the quality walks vs. all of the walks, but conveys the constraints on walk length due to the floor plan of the apartment. Furthermore, the number of quality walks compared to the number of all walks illustrates the issues with capturing quality walks, such as occlusion, or simply lack of good walking paths in the apartment. Density plot 2(c) indicates a tight, single cluster formed in the 2D feature space of height and speed. Finally, Figure 2(d) illustrates the paths of the extracted walks in the apartment.

To obtain measures of habitual, in-home speed, stride time, and stride length for the resident, a single 4D Gaussian distribution was fit to the data from the quality walks. The 4dimensions were: [average speed, stride time, stride length, height]). Final mean values of the parameters for the resident are shown in Table I.

In order to verify that these values were indeed computed from walks of the resident and to evaluate the impact of 
visitor walks on the automatically estimated parameters, a person familiar with the resident hand labeled approximately one third of the quality walks from the apartment; which were selected randomly. (For the other apartments, if this resulted in less than 95 labeled walks, the percentage was increased). This labeling was achieved by viewing videos of the Kinect depth imagery of the walks. Although it cannot be guaranteed that these labels are 100 percent accurate, someone familiar with the resident is able to label the Kinect video of a walk with high confidence.

The results of this hand labeling are provided in Table I. First, $98 \%$ of the labeled walks in Apartment 1 were from the resident. Furthermore, $100 \%$ of the labeled walks used in the automated estimate of average speed, average stride time, average stride length, and height were from the resident. This provides persuasive evidence that the measurements obtained for this resident are accurate and not significantly impacted by visitor walks in the apartment. Finally, Table I contains gait parameters for the resident computed solely from the labeled quality walks (shown in brackets [ ]) for comparison purposes.

\section{B. Apartment $2 / 3$}

Apartments 2 and 3 were processed in the same manner as Apartment 1 and the gait measurement results are shown in Table I. Although the residents in Apartment 2 and 3 both used a walker for all or part of the three week period, the system was still able to extract the parameters of stride time and stride length for a number of the identified walks. The large difference in the total number of walks and quality
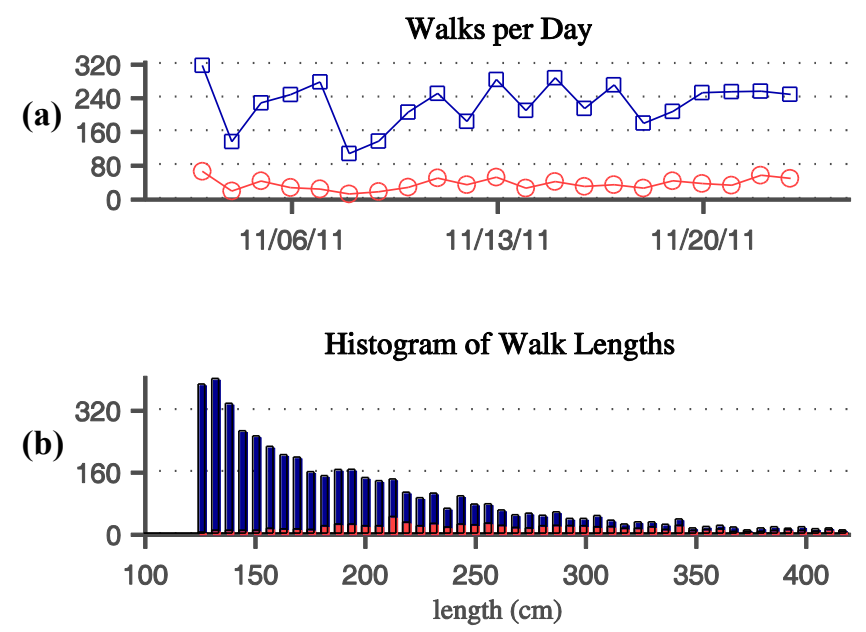

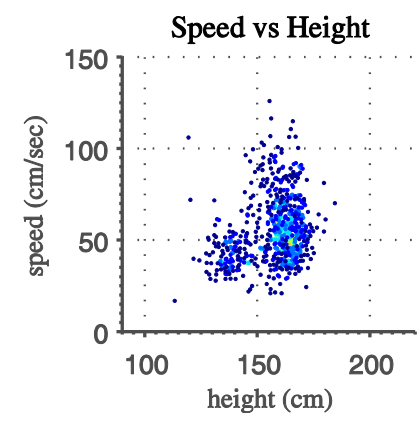

(c)

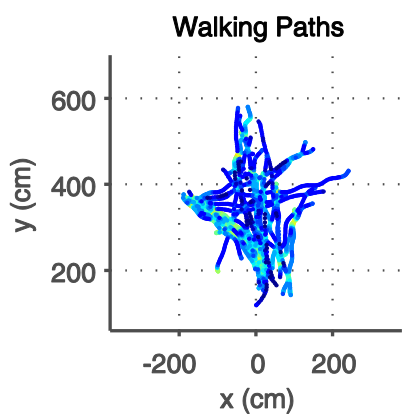

(d)
Figure 3: Data from Apartment 4. (a) Plot of the number of identified walks per day: all (blue square), quality (red circle). (b) Histogram of the walk lengths: all (blue), quality (red). (c) Density plot of speed vs. height for the quality walks. (d) Density plot of the paths of the quality walks.

\begin{tabular}{|c|c|c|c|c|c|c|c|}
\hline \multicolumn{8}{|c|}{$\begin{array}{c}\text { TABLE I } \\
\text { SUMMARY OF IN-HOME DATA AND MEASUREMENTS FROM KINECT SYSTEMS } \\
\left.\text { NOVEMBER } 3^{R D}-\text { NOVEMBER } 23^{R D}, 2011 \text { (21 DAYS }\right)\end{array}$} \\
\hline & APART. 1 & APART. 2 & APART. 3 & \multicolumn{2}{|c|}{ APARTMENT 4} & \multicolumn{2}{|c|}{ APARTMENT 5} \\
\hline Number of walks identified & 1140 & 1721 & 2255 & \multicolumn{2}{|c|}{4700} & \multicolumn{2}{|c|}{596} \\
\hline Number of quality walks identified & 297 & 136 & 392 & \multicolumn{2}{|c|}{699} & \multicolumn{2}{|c|}{39} \\
\hline Percentage of labeled walks from Resident 1 & 98.0 & 81.8 & 93.9 & \multicolumn{2}{|c|}{69.1} & \multicolumn{2}{|c|}{44.7} \\
\hline Percentage of labeled walks from Resident 2 & - & - & - & \multicolumn{2}{|c|}{21.0} & \multicolumn{2}{|c|}{50.0} \\
\hline \multirow[t]{2}{*}{ Percentage of labeled walks from visitor } & 2.0 & 18.2 & 6.1 & \multicolumn{2}{|c|}{9.9} & \multicolumn{2}{|c|}{5.3} \\
\hline & RES. 1 & RES. 1 & RES. 1 & RES. 1 & RES. 2 & RES. 1 & RES. 2 \\
\hline $\begin{array}{l}\text { Number of quality walks used in estimate } \\
\quad \text { Automated [Computed using labeled walks] }\end{array}$ & $\begin{array}{l}224 \\
{[98]} \\
\end{array}$ & $\begin{array}{c}94 \\
{[81]} \\
\end{array}$ & $\begin{array}{c}290 \\
{[123]}\end{array}$ & $\begin{array}{c}363 \\
{[161]} \\
\end{array}$ & $\begin{array}{c}129 \\
{[49]} \\
\end{array}$ & $\begin{array}{c}3 \\
{[17]} \\
\end{array}$ & $\begin{array}{c}21 \\
{[19]} \\
\end{array}$ \\
\hline $\begin{array}{c}\text { Percentage of labeled walks used from resident } \\
\text { Automated [Computed using labeled walks] }\end{array}$ & $\begin{array}{c}100 \\
{[100]}\end{array}$ & $\begin{array}{c}100 \\
{[100]}\end{array}$ & $\begin{array}{c}100 \\
{[100]}\end{array}$ & $\begin{array}{l}94.6 \\
{[100]}\end{array}$ & $\begin{array}{c}97.7 \\
{[100]}\end{array}$ & $\begin{array}{c}100 \\
{[100]}\end{array}$ & $\begin{array}{c}35.0 \\
{[100]}\end{array}$ \\
\hline $\begin{array}{l}\text { Average speed(cm/sec) } \\
\quad \text { Automated [Computed using labeled walks] }\end{array}$ & $\begin{array}{c}62.2 \\
{[61.0]}\end{array}$ & $\begin{array}{c}30.5 \\
{[29.7]}\end{array}$ & $\begin{array}{c}40.1 \\
{[39.4]}\end{array}$ & $\begin{array}{c}57.2 \\
{[58.4]}\end{array}$ & $\begin{array}{c}44.5 \\
{[42.7]}\end{array}$ & $\begin{array}{c}50.5 \\
{[40.4]}\end{array}$ & $\begin{array}{c}33.0 \\
{[31.2]}\end{array}$ \\
\hline $\begin{array}{l}\text { Average stride time(sec) } \\
\quad \text { Automated [Computed using labeled walks] }\end{array}$ & $\begin{array}{c}1.17 \\
{[1.17]}\end{array}$ & $\begin{array}{c}2.03 \\
{[2.06]}\end{array}$ & $\begin{array}{c}1.46 \\
{[1.47]}\end{array}$ & $\begin{array}{c}1.50 \\
{[1.49]}\end{array}$ & $\begin{array}{c}1.31 \\
{[1.34]}\end{array}$ & $\begin{array}{c}1.26 \\
{[1.44]}\end{array}$ & $\begin{array}{c}1.62 \\
{[1.64]}\end{array}$ \\
\hline $\begin{array}{l}\text { Average stride length(cm) } \\
\quad \text { Automated [Computed using labeled walks] }\end{array}$ & $\begin{array}{c}71.6 \\
{[70.1]}\end{array}$ & $\begin{array}{c}59.9 \\
{[59.7]}\end{array}$ & $\begin{array}{c}57.2 \\
{[56.1]}\end{array}$ & $\begin{array}{c}83.1 \\
{[83.8]}\end{array}$ & $\begin{array}{c}56.9 \\
{[55.9]}\end{array}$ & $\begin{array}{c}63.2 \\
{[56.4]}\end{array}$ & $\begin{array}{c}52.3 \\
{[51.6]}\end{array}$ \\
\hline $\begin{array}{l}\text { Height(cm) } \\
\quad \text { Automated [Computed using labeled walks] }\end{array}$ & $\begin{array}{c}162.1 \\
{[161.8]}\end{array}$ & $\begin{array}{c}156.5 \\
{[156.5]}\end{array}$ & $\begin{array}{c}140.7 \\
{[139.4]}\end{array}$ & $\begin{array}{c}162.6 \\
{[162.1]}\end{array}$ & $\begin{array}{c}137.9 \\
{[136.7]}\end{array}$ & $\begin{array}{c}154.9 \\
{[157.7]}\end{array}$ & $\begin{array}{c}154.4 \\
{[153.7]}\end{array}$ \\
\hline
\end{tabular}


walks per day between the three apartments reflects the impact of a variety of factors involving both the residents themselves and their environments. Finally, although almost a fifth of the walks in Apartment 2 were from visitors, these did not significantly change the gait parameter estimates.

\section{Apartment 4}

Figure 3 provides visualizations of the walk data obtained from Apartment 4, which had two residents. Density plot 3(c) shows two clusters formed in the 2D feature space of height and speed. In a similar manner to Apartment 1, a 4D Gaussian Mixture Model (GMM) with the number of distributions set to two was fit to the data from the quality walks. Final mean values are shown in Table I. (In the multiple resident apartments, the manually measured heights of the residents were used to match them to the distributions.)

As with the other apartments, a random sampling of the quality walks were hand labeled by someone familiar with the residents. Approximately 95 percent of the labeled walks used in the automated estimate for Resident 1 were actually from Resident 1, and approximately 98 percent of the labeled walks used for Resident 2 were actually from Resident 2, even as Resident 2 was responsible for only 21 percent of all the labeled walks in the apartment.

\section{Apartment 5}

Although Apartment 5 had two residents, the data from the apartment forms a single, sparse cluster. This is a result of three factors. First, the residents are very similar in height and functional ability. Second, the residents live a rather sedentary life style. Third, the apartment contains a single viable walking path that is slightly curved and suffers from occlusion issues. As a result, the number of identified walks in the apartment is greatly reduced and the walks that are identified are quite similar in the 4D space.

Despite the limitations of the data from Apartment 5, a 4D GMM with the number of distributions set to two was fit to the data from the quality walks. As the results in Table I indicate, the GMM is essentially unable to identify two separate distributions and, thus, one distribution is modeled on the majority of the data points while the other is modeled on a small group of relative outliers. Although this small group of outliers does consist of walks from the correct resident, they are not representative of the entire data set, as illustrated by a comparison against the parameter estimates computed from the labeled walks.

\section{Discussion}

As shown in Section III, Kinect systems deployed in the apartments of elderly residents in an independent living facility were able to unobtrusively identify walking sequences and automatically generate habitual, in-home gait parameter estimates for the residents. This was achieved in both one and two resident homes and in the presence of visitors. Furthermore, the stride parameters of time and length were obtained from walking sequences of residents using a walker.
Analysis of the data indicated that a methodology of fitting a GMM with the number of distributions equal to the number of residents was successful in filtering out the majority of non-resident walking sequences, allowing accurate gait parameter estimates to be made. In future work, mode finding algorithms, which may be less sensitive to outliers, will also be investigated for this purpose.

The potential cost of the systems is relatively minimal. The Kinect sensor currently retails for $\$ 150$ and a suitable computer can also be obtained inexpensively. Current goals and future work include developing a system for generating automatic fall risk assessments, as well as evaluating the usefulness of different in-home gait parameters in detecting early signs of illness.

\section{ACKNOWLEDGMENT}

This work was supported in part by the U.S. National Science Foundation under grants CNS-0931607 and IIS0703692 and by the US Agency for Healthcare Research and Quality under grant R01-HS018477.

\section{REFERENCES}

[1] D. Hodgins, "The Importance of Measuring Human Gait," Medical Device Technology, 2008, 19(5):42, 44-7.

[2] M. Runge and G. Hunter, "Determinants of musculoskeletal frailty and the risk of falls in old age," Journal of Musculoskeletal and Neuronal Interactions, 6 (2006), 167-173.

[3] BE. Maki, Gait changes in older adults: predictors of falls or indicators of fear, Journal of the American Geriatrics Society, vol. 45(3), pp. 313-20, 1997.

[4] JM Hausdorff, DA Rios, HK Edelberg, "Gait variability and fall risk in community-living older adults: a 1-year prospective study," Arch Phys Med Rehabilitation, 2001, 82:1050-6.

[5] Y. Barak et al., "Gait Characteristics of Elderly People with a History of Falls: A Dynamic Approach," Phys Ther., 86(11): 1501-10 2006.

[6] M. Montero-Odasso, M. Schapira, E.R. Soriano, M. Varela,R. Kaplan, L.A. Camera and L.M. Mayorga, "Gait velocity as a single predictor of adverse events in healthy seniors aged 75 years and older," J. of Gerontol. A Biol. Sci. Med. Sci. 60 (2005), 1304-1309.

[7] R. Camicioli, D. Howieson, B. Oken, G. Sexton and J. Kaye, "Motor slowing precedes cognitive impairment in the oldest old," Neurology 50 (1998), 1496-1498.

[8] J. Kaye, N. Mattek, H. Dodge, T. Buracchio, D. Austin, S. Hagler, M. Pavel, and T. Hayes, "One walk a year to 1000 within a year: Continous in-home unobtrusive gait assessment of older adults," Gait and Posture, 35(2), pp. 197-202, 2012.

[9] S. Hagler et al., "Unobtrusive and Ubiquitous In-Home Monitoring: A Methodology for Continuous Assessment of Gait Velocity in Elders," IEEE Trans Biomed Eng, 2009.

[10] B.R. Greene, A. O'Donovan, R. Romero-Ortuno, L. Cogan, C. N. Scanaill, and R.A. Kenny, "Quantitative Falls Risk Assessment Using the Timed Up and Go Test," IEEE Trans. on Biomedical Engineering, vol. 57, no. 12, Dec., 2010, pp. 2918-2926.

[11] E. Stone and M. Skubic, "Evaluation of an Inexpensive Depth Camera for In-Home Gait Assessment," Journal of Ambient Intelligence and Smart Environments, 3(4):349-361, 2011.

[12] E. Stone, D. Anderson, M. Skubic, and J. Keller, "Extracting Footfalls from Voxel Data," 32nd Annual International Conference of the IEEE Engineering in Medicine and Biology Society, Buenos Aires, Argentina, Aug 31-Sep 4, 2010.

[13] G. Demiris, M. Rantz, M. Aud, K. Marek, H. Tyrer, M. Skubic, and A. Hussam, "Older Adults' Attitudes Towards and Perceptions of 'Smarthome' Technologies: a Pilot Study," Medical Informatics and The Internet in Medicine, June, 2004, vol. 29, no. 2, pp. 87-94.

[14] G. Demiris et al., "Older adults' privacy considerations for vision based recognition methods of eldercare applications," Technology and Health Care, vol. 17, pp. 41- 48, 2009. 